\title{
Evolution of properties of macadamia husk throughout gasification: hints for a zero-waste energy production system
}

\author{
Linh $\mathrm{Vu}^{1}$, Dinh Nguyen ${ }^{1}$, Dong Nguyen ${ }^{1}$, Bay Tran ${ }^{2}$, Hoai Nguyen ${ }^{3}$, Nga Mai ${ }^{4}$, and Nam \\ Nguyen $^{5}$ \\ ${ }^{1}$ VNU University of Engineering and Technology Faculty of Engineering Physics and \\ Nanotechnology \\ ${ }^{2}$ University of Transport and Communication \\ ${ }^{3}$ Hanoi University of Science \\ ${ }^{4}$ Faculty of Environmental and Natural Resources, Ha Tay Community College \\ ${ }^{5}$ University of Science and Technology of Hanoi
}

October 28,2021

\begin{abstract}
In-depth information about the transformation of biomass during gasification is the key to the sustainable development of this technology. This study elucidated the evolution of physico-chemical properties of macadamia husk throughout relevant industrial gasification conditions. The technical characteristics combined with high kinetics highlighted the suitability of this feedstock for gasification. Non-porous chars with very low surface areas were observed throughout the conversion, which minimizes the potential to use the residues after gasification as adsorbents. Nevertheless, multiple carboxyl and hydroxyl functional groups were present on the char surface. Moreover, an extraordinarily high K content was detected (up to 86 wt\% of the char's total inorganic elements) and evenly distributed on the char surface. Such results consolidated the idea of combining the production of energy and cheap bio-fertilizers using the gasification of macadamia husk. The resulting database offered interesting hints for the development of zero-waste energy production systems with biomass gasification.
\end{abstract}

\section{Hosted file}

AIche_MH biochar evolution of properties_Final.docx available at https://authorea.com/ users/443470/articles/543465-evolution-of-properties-of-macadamia-husk-throughoutgasification-hints-for-a-zero-waste-energy-production-system

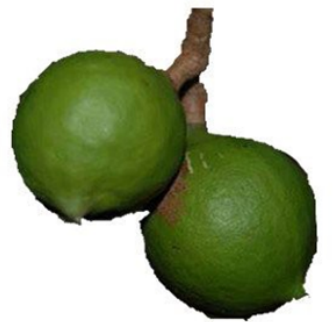

(a)
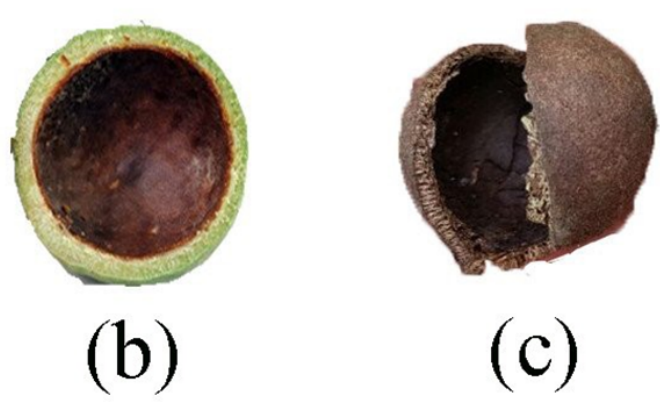
figures/char-coversion-kinetics/char-coversion-kinetics-eps-converted-to.pdf
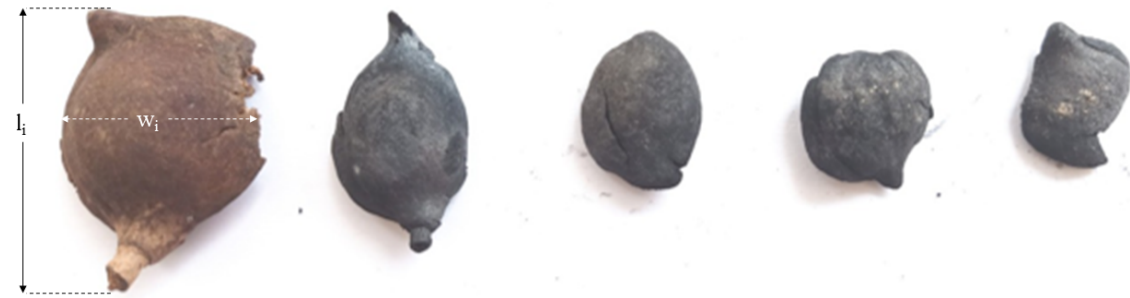

Raw MH

$\mathrm{X}=0$

$\mathrm{X}=0.25$

$\mathrm{X}=0.5$

$X=0.75$ 
figures/N2-adsorption-desorption/N2-adsorption-desorption-eps-converted-to.pdf 
figures/FTIR-spectra/FTIR-spectra-eps-converted-to.pdf 
figures/XRF-spectra/XRF-spectra-eps-converted-to.pdf

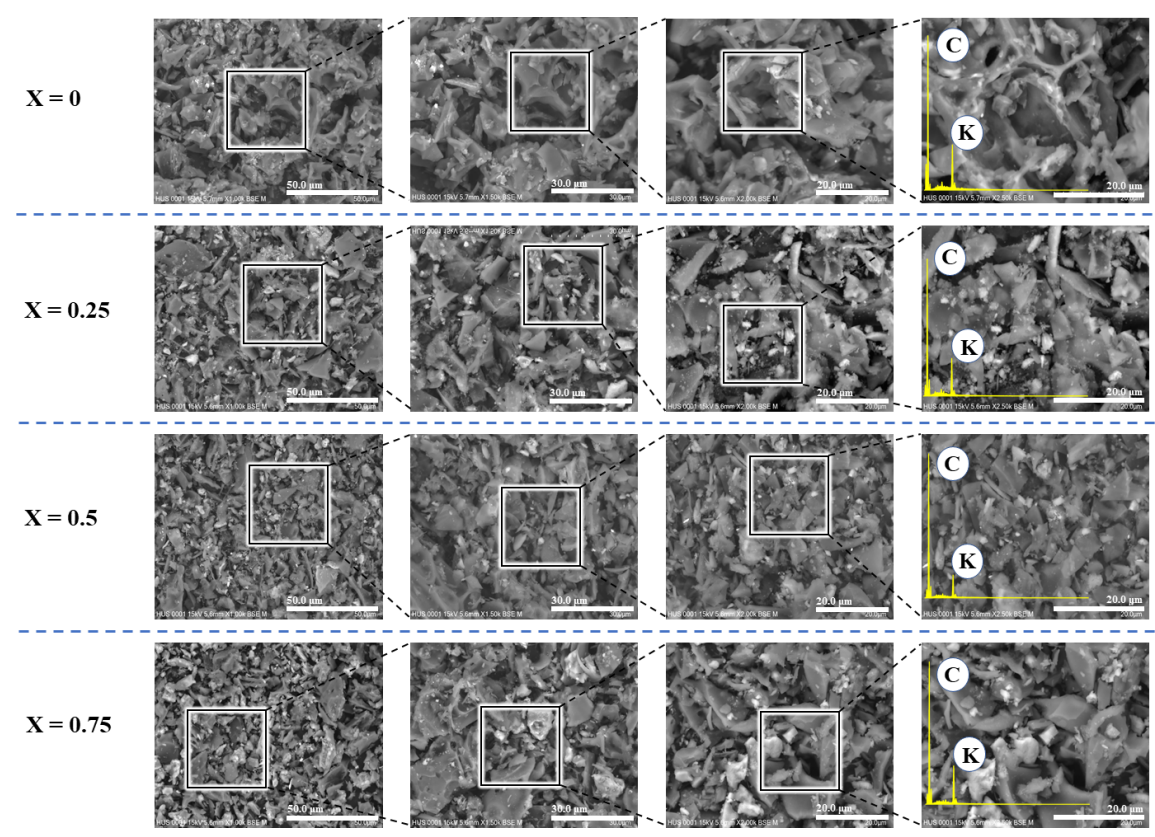

